\title{
Plasma response to a single dose of dietary $\beta$-cryptoxanthin esters from papaya (Carica papaya L.) or non-esterified $\beta$-cryptoxanthin in adult human subjects: a comparative study
}

\author{
Dietmar E. Breithaupt ${ }^{1 \star}$, Philipp Weller ${ }^{1}$, Maike Wolters ${ }^{2}$ and Andreas Hahn ${ }^{2}$ \\ ${ }^{1}$ Institute of Food Chemistry, University of Hohenheim, Garbenstr. 28, 70593 Stuttgart, Germany \\ ${ }^{2}$ Institute of Food Science, University of Hannover, Wunstorferstr. 14, 30453 Hannover, Germany
}

(Received 17 December 2002 - Revised 22 May 2003 - Accepted 23 June 2003)

\begin{abstract}
Many orange-coloured fruits contain $\beta$-cryptoxanthin in its non-esterified as well as its esterified form. Information concerning the absorption of $\beta$-cryptoxanthin, especially with regard to the metabolism of its fatty acid esters, is rather scarce. The present study assessed the plasma concentration reached after consumption of a single dose of native $\beta$-cryptoxanthin esters from papaya (Carica papaya L.) or nonesterified $\beta$-cryptoxanthin in equal total amounts. In a randomized, single-blind crossover study, twelve subjects were served a portion of yoghurt containing esterified or non-esterified $\beta$-cryptoxanthin $(1.3 \mathrm{mg}$ absolute) together with a balanced breakfast. Between the two intervention days, there was a 2-week depletion period. After a fasting blood sample had been taken, futher samples were taken from the subjects at 3, 6, 9, 12 and $24 \mathrm{~h}$. The concentration of non-esterified $\beta$-cryptoxanthin in the whole plasma was determined by HPLC; $\beta$-cryptoxanthin identification was confirmed by liquid chromatography-atmospheric pressure chemical ionization-MS analyses. Irrespective of the consumed diet, the plasma $\beta$-cryptoxanthin concentrations increased significantly $(P=0 \cdot 05)$ and peaked after $6-12 \mathrm{~h}$. The concentration curves, as well as the areas under the curves, were not distinguishable according to two-sided $F$ and $t$ tests $(P=0 \cdot 05)$. Standardization of $\beta$-cryptoxanthin concentrations to plasma triacylglycerol and cholesterol had no impact on the results. Thus, the present study indicates comparable bioavailability of both non-esterified $\beta$-cryptoxanthin and mixtures of $\beta$-cryptoxanthin esters. The results support the existence of an effective enzymatic cleavage system accepting various $\beta$-cryptoxanthin esters.
\end{abstract}

$\beta$-Cryptoxanthin: Carotenoid ester: Plasma carotenoid response: Liquid chromatography-atmospheric pressure chemical ionization-mass spectroscopy

About 600 naturally occurring carotenoids have been identified. About twenty of these compounds have been detected in mammal plasma and tissues, with $\beta$-carotene, lycopene, lutein, $\beta$-cryptoxanthin and $\alpha$-carotene accounting for $>90 \%$ of the circulating carotenoids in human plasma (Bieri et al. 1985; Rock, 1997). With regard to provitamin A activity, only $\alpha$-carotene, $\beta$-carotene and $\beta$-cryptoxanthin play an important role in the daily diet. Noteworthy in this context is $\beta$-cryptoxanthin, a xanthophyll with only one of its ionone-rings hydroxylated, and therefore possessing provitamin A activity. Although $\beta$-cryptoxanthin only occurs as a minor carotenoid in the majority of fruits and vegetables, the concentration of this micronutrient in the serum can be significantly greater than that of $\alpha$-carotene, depending on the diet (Stahl et al. 1992). $\beta$-Cryptoxanthin occurs mainly in orange-coloured fruits such as papaya, orange, apricot, mandarin, peach, loquat and red pepper. Especially with regard to the high consumption of oranges and mandarins in some populations, those fruits are regarded as valuable $\beta$-cryptoxanthin sources, whereas the consumption of loquat, peach or apricot with an equally high $\beta$-cryptoxanthin content is seasonally dependent. The occurrence, however, is not limited only to non-esterified $\beta$-cryptoxanthin: a various number of fatty acid esters are common, mainly laurate, myristate and palmitate (Wingerath et al. 1995; Breithaupt \& Bamedi, 2001).

Although extensive feeding studies have been conducted to evaluate absorption, distribution and metabolism of $\beta$-carotene (van Vliet et al. 1995) and lycopene (Gärtner et al. 1997) in human subjects, little information is available concerning $\beta$-cryptoxanthin, particularly its esters. While recent studies have dealt with the metabolism of $\beta$-cryptoxanthin in freshwater fish (Goswami, 1984), others have focused on the metabolism and biological potency in the rat, with special emphasis on the formation of retinal (John et al. 1970). Although several studies on human subjects have quantified $\beta$-cryptoxanthin, together with other fat-soluble micronutrients, in plasma or tissue (Thurnham, 1988; Ito et al. 1990; Krinsky et al. 1990; Ross et al. 1995; Olmedilla et al. 1997; Grobusch-Klipstein et al. 2000), no comparative study concerning the

Abbreviations: APcI, atmospheric pressure chemical ionization; AUC, area under the curve; LC, liquid chromatography; MBTE, methyl-tert-butyl ether.

* Corresponding author: Dr Dietmar E. Breithaupt, fax +49 711 4594096, email breithau@uni-hohenheim.de 
absorption efficiency of esterified and non-esterified $\beta$-cryptoxanthin yet exists.

It is generally assumed that carotenoid esters are hydrolysed before or during absorption and that the non-esterified form is absorbed, as in the case of other esterified isoprenoids (e.g. cholesteryl esters). Wingerath et al. (1995) investigated the carotenoid pattern in chylomicrons and serum after ingestion of concentrated tangerine juice rich in different $\beta$-cryptoxanthin esters. They observed increasing amounts of non-esterified $\beta$-cryptoxanthin in chylomicrons as well as serum, but no $\beta$-cryptoxanthin esters were detectable. This indicates a cleavage in the intestine before incorporation into lipoproteins. To our knowledge, up to now no study has compared the plasma concentrations of $\beta$-cryptoxanthin after ingestion of esterified and non-esterified $\beta$-cryptoxanthin using plasma HPLC analyses. Since non-esterified $\beta$-cryptoxanthin is the only form found in human plasma, it is essential to include non-esterified $\beta$-cryptoxanthin as reference compound in a comparative study.

Since the present study began with a 2-week depletion phase, the plasma concentration of $\beta$-cryptoxanthin was expected to be significantly reduced. The base concentration of endogenous $\beta$-cryptoxanthin was therefore low enough to allow the use of whole plasma and not of chylomicrons, as was the case in recent investigations when studying intestinal absorption of carotenoids (van Vliet et al. 1995; Wingerath et al. 1995). Since Wingerath et al. (1995) showed that $\beta$-cryptoxanthin esters appeared neither in plasma nor in chylomicrons, the whole plasma can be used without investigating the compartments separately. Consequently, we determined the plasma concentration curves of $\beta$-cryptoxanthin using the whole plasma after a single oral dose of esterified or non-esterified $\beta$-cryptoxanthin on two occasions, 2 weeks apart.

Since $\beta$-cryptoxanthin is yet not available as a food additive, we used papaya (Carica papaya L.) puree to obtain native $\beta$-cryptoxanthin esters. As well as $\beta$-cryptoxanthin, several minor carotenoids, such as lutein, lycopene and others, occur in this tropical fruit. Although the $\beta$-cryptoxanthin ester pattern of papaya was reported earlier by our group (Breithaupt et al. 2002), the ester composition of the puree used in the present study was determined by liquid chromatography (LC)-atmospheric pressure chemical ionization (APcI)-MS analysis. Non-esterified $\beta$-cryptoxanthin was obtained from the same plant food source after saponification of the crude oil extract, with two subsequent clean-up steps. For application, both forms were dissolved in sunflower oil and dispersed into yoghurt, served together with a balanced breakfast. Thus, possible effects of the plant food matrix, which may influence the absorption of carotenoids, can be excluded. Using similar amounts of total $\beta$-cryptoxanthin in the final diet allowed us to compare directly the resulting plasma concentrations. The absolute amount present in both diets was set to $1.3 \mathrm{mg} \beta$-cryptoxanthin, corresponding to a serving size of about $100 \mathrm{~g}$ native papaya, a quantity easily consumable within the conventional daily diet. Thus, a physiological dose of total $\beta$-cryptoxanthin was administered to the subjects.

\section{Materials and methods}

\section{Materials}

$\beta$-Cryptoxanthin as a reference compound was generously provided by Hoffmann-LaRoche (Basel, Switzerland). Acetone, 2,6-di-tert-butyl-4-methylphenol, diethyl ether, ethyl acetate, $n$-hexane, light petroleum $\left(40-60^{\circ} \mathrm{C}\right)$, ethanol and methanol were purchased from Merck (Darmstadt, Germany). $\beta$-apo-12'-Carotenal was provided by BASF (Ludwigshafen, Germany), and methyl-tertbutyl ether (MTBE) and $\beta$-carotene were obtained from Fluka (Neu-Ulm, Germany). Test kits for in vitro determination of plasma triacylglycerol (method 334-UV) and total cholesterol (method 401) were from Sigma (Taufkirchen, Germany). All solvents were of analytical grade and were distilled before use. For HPLC, ultra-pure water obtained from a Milli-Q 185 apparatus (Millipore, Eschborn, Germany) was employed. The papaya puree was a gift from Schutzgemeinschaft der Fruchtsaft-Industrie e.V. (Nieder-Olm, Germany) and was stored frozen at $-20^{\circ} \mathrm{C}$ until processing.

\section{Subjects}

Twelve human subjects, recruited from staff and students of the Institute of Food Science (Hannover, Germany), six female and six male, were divided at random into two groups of six each. Four of the subjects were smokers. Three of the female subjects used contraceptives. None of the subjects suffered from gastrointestinal diseases, or took laxatives or drugs lowering plasma triacylglycerol or cholesterol concentrations. They did not take any supplements containing carotenoids or vitamins in the 8 weeks before and during the study. Subjects were requested to keep to a normal diet, but to avoid $\beta$-cryptoxanthin-rich fruit such as peaches, oranges, papaya, apricots and red peppers for the 2 weeks before and the 2 weeks during the intervention period. Products manufactured from these plant sources (e.g. orange juice, dried apricots, vitamin-fortified mixed fruit beverages) were also to be avoided. The study protocol was approved by the Medical Ethics Committee of the Medizinische Hochschule, Hannover, and the participants provided written informed consent.

\section{Study design}

The study was designed as a single-blind crossover study for $16 \mathrm{~d}$ and consisted of two experimental days, separated by a 2 -week depletion period, during which no supplementation was carried out. On each of the $2 \mathrm{~d}$, subjects received a balanced breakfast with the respective supplementation (see Table 1). For the two experimental days, subjects were instructed not to consume any fruits or products made from fruits, including juices. Carotenoid supplementation was performed by mixing a serving of $150 \mathrm{~g}$ commercial whole-milk yoghurt $(35 \mathrm{~g}$ fat $/ \mathrm{kg})$ with $4 \mathrm{~g}$ spiked sunflower oil, resulting in a total of $1.3 \mathrm{mg} \beta$-cryptoxanthin in either the esterified or non-esterified form. The yoghurt was consumed together with a standardized breakfast, with a total of approximately $24 \mathrm{~g}$ fat and $2100 \mathrm{~kJ}$ 
Table 1. Design of the single-blind crossover study*

\begin{tabular}{llc}
\hline Group & \multicolumn{1}{c}{ Day 1 } & Day 2 \\
\hline Group 1 $(n 6)$ & $\beta$-Cryptoxanthin ester & $\begin{array}{c}\text { Non-esterified } \\
\beta \text { cryptoxanthin }\end{array}$ \\
Group 2 $(n 6)$ & $\begin{array}{c}\text { Non-esterified } \\
\beta \text { cryptoxanthin }\end{array}$ & $\beta$-Cryptoxanthin ester \\
& & \\
\hline
\end{tabular}

* Subjects were divided at random into two groups; the intervention was carried out on two days 2 weeks apart.

(about $500 \mathrm{kcal}$; see Table 2). Blood samples were collected before $(0 \mathrm{~h})$ and after supplementation $(3,6,9$, 12 and $24 \mathrm{~h}$ ). After $24 \mathrm{~h}$, participants were again instructed to consume a low $\beta$-cryptoxanthin diet for another 2 weeks to allow for depletion. After 2 weeks, the crossover was performed.

\section{Preparation of supplements}

Extraction of carotenoids. Carotenoids were extracted from papaya puree using a ternary solvent mixture of light petroleum $\left(40-60^{\circ} \mathrm{C}\right)$-ethyl acetate-methanol (1:1:1, by vol.). A sample of the puree $(500 \mathrm{~g})$ was transferred into a 2 litre flask and extracted with $500 \mathrm{ml}$ solvent by mixing thoroughly. The extract was separated from the plant tissue employing a Büchner funnel. To allow for complete extraction, portions of $200 \mathrm{ml}$ solvent were poured on to the filter cake and sucked off. This step was repeated until the filter cake was nearly colourless. The resulting extract was transferred into a separating funnel and washed with water containing a saturated solution of $\mathrm{NaCl}$ to allow for a good phase separation. The water layer was discarded, while the organic layer was transferred into an Erlenmeyer flask, dried with anhydrous sodium sulfate, filtered, and evaporated to dryness. The resulting oily, orange residue was redissolved in light petroleum $\left(40-60^{\circ} \mathrm{C}\right)$ and stored at $-20^{\circ} \mathrm{C}$.

Isolation of non-esterified $\beta$-cryptoxanthin. To obtain non-esterified $\beta$-cryptoxanthin, the crude oil was saponified using methanolic $\mathrm{KOH}$ (300 g/l methanol). Therefore, the solution obtained as described earlier was evaporated to dryness and the remaining oil dissolved in $100 \mathrm{ml}$ diethyl ether. After the addition of methanolic $\mathrm{KOH}(5 \mathrm{ml})$, the mixture was kept at room temperature for $12 \mathrm{~h}$, protected from light. The diethyl ether phase was washed twice with water, dried with sodium sulfate and filtered. To separate all-trans- $\beta$-cryptoxanthin from cis-isomers,

Table 2. Composition of the standardized breakfast*

\begin{tabular}{lccc}
\hline & Quantity (g) & Fat (g) & Energy (kJ) \\
\hline Bread roll & $40 \cdot 0$ & 0.7 & 462 \\
Wholegrain bread & $30 \cdot 0$ & 0.3 & 245 \\
Butter & $10 \cdot 0$ & $8 \cdot 3$ & 309 \\
Cream cheese $320 \mathrm{~g} \mathrm{fat} / \mathrm{kg}$ & $16 \cdot 7$ & $5 \cdot 3$ & 235 \\
Full-fat yoghurt $35 \mathrm{~g} \mathrm{fat} / \mathrm{kg}$ & $150 \cdot 0$ & $5 \cdot 3$ & 440 \\
Oil suspension & 4.0 & 4.0 & 148 \\
Honey & 20.0 & - & 257 \\
Total & & 23.9 & 2096 \\
\hline
\end{tabular}

*Values were calculated from food composition tables (Scherz et al. 2000). semi-preparative HPLC was employed using an isocratic solvent system (methanol-MTBE (57:43, v/v); retention time of the all-trans-isomer $5.5 \mathrm{~min}$ ). Instantly after isolation, the combined eluates from multiple separations were evaporated under reduced pressure and dim light. For storage, the now crystalline $\beta$-cryptoxanthin was redissolved in light petroleum $\left(40-60^{\circ} \mathrm{C}\right)$ and was frozen at $-20^{\circ} \mathrm{C}$ in a $\mathrm{N}_{2}$-filled flask. For supplementation, light petroleum $\left(40-60^{\circ} \mathrm{C}\right)$ was removed and $\beta$-cryptoxanthin was dissolved in sunflower oil. The concentration of the xanthophyll was adjusted to 321 (SD10) $\mu \mathrm{g} / \mathrm{g}$ ( $n$ 3) by dilution with oil. Using $4 \mathrm{~g}$ oil per diet, this corresponds to an intake of $1.3 \mathrm{mg}$ non-esterified $\beta$-cryptoxanthin (see p. 796).

Clean-up of native $\beta$-cryptoxanthin esters. For cleanup, a chromatographic glass column $(300 \times 20 \mathrm{~mm})$ was packed with silica gel $60(0.063-0.200 \mathrm{~mm})$, suspended in light petroleum $\left(40-60^{\circ} \mathrm{C}\right)$, to approximately $100 \mathrm{~mm}$ height. The light petroleum solution resulting from a work-up of $500 \mathrm{~g}$ papaya puree was poured carefully onto the column, allowing the solvent and apolar compounds to run through. To elute carotenoids, mixtures of acetone in light petroleum were used. At $20 \mathrm{ml}$ acetone/l, lycopene and the major portion of $\beta$-carotene were eluted from the column, together with other lipid constituents; $\beta$-cryptoxanthin esters were obtained with $20-40 \mathrm{ml}$ acetone/l. More polar carotenoids (e.g. non-esterified $\beta$-cryptoxanthin and lutein) remained on the column and were discarded. Fractions containing $\beta$-cryptoxanthin esters were evaporated to dryness and redissolved in light petroleum. For supplementation, the solvent was removed and $\beta$-cryptoxanthin esters were dissolved in sunflower oil. The concentration of xanthophyll was set to 319 (SD 19) $\mu \mathrm{g} / \mathrm{g}$ oil $(n 3$; determined after saponification) by dilution with oil. Using $4 \mathrm{~g}$ oil per diet, this results in an intake of $1.3 \mathrm{mg}$ $\beta$-cryptoxanthin in esterified form. For LC-APcI-MS analyses of native $\beta$-cryptoxanthin esters, the residue of one additional preparation was dissolved in methanolMTBE (1:1, v/v). The stability of the respective carotenoids in sunflower oil and in the yoghurt was tested at the end of the study. After 2 weeks storage at $-20^{\circ} \mathrm{C}$, both spiked oils showed neither degradation nor isomerization $(<1 \%$ compared with the initial content); likewise, carotenoid esters were stable in the yoghurt.

Plasma sample preparation. At any blood withdrawal, about $5 \mathrm{ml}$ was sampled from each participant. Immediately after collection, the plasma was separated from the erythrocytes by centrifugation $(5000 \mathrm{rpm}, 10 \mathrm{~min})$ and stored at $-20^{\circ} \mathrm{C}$ in plastic caps. The work-up procedure is based on a method described by Khachik et al. (1997). In brief, a sample of the plasma $(0.75-1.00 \mathrm{ml})$ was precipitated with ethanol $(1 \mathrm{ml}$, containing $10 \mathrm{~g}$ butylated hydroxytoluene/l) and mixed vigorously on a shaker (Vortex Genie 2; Scientific Industries, Bohemia, NY, USA). A portion $(1 \mathrm{ml})$ of the internal standard $(2 \mu \mathrm{g}$ $\beta$-apo- $12^{\prime}$-carotenal/l ethanol) was added and the solution mixed again. To extract the carotenoids, $2 \mathrm{ml} n$-hexane were added to the homogenate, the mixture stirred once again and the precipitate spun down in a Biofuge $\mathrm{R}$ centrifuge (Heraeus, Hanau, Germany) at $644 \mathrm{~g}$ at $10^{\circ} \mathrm{C}$ for $5 \mathrm{~min}$. The organic layer was transferred into a $10 \mathrm{ml}$ brown glass vial and the extraction procedure was 
repeated. The combined organic phases were evaporated and redissolved in $1 \mathrm{ml}$ methanol-MTBE $(1: 1, \mathrm{v} / \mathrm{v})$. The solution obtained was passed through a $0.45 \mu \mathrm{m}$ membrane filter and was used immediately for HPLC analysis. All steps were performed under dim light. To determine possible losses during the work-up procedure, the HPLC peak areas of the internal standard, $\beta$-apo- $12^{\prime}$-carotenal, were monitored. The recovery of $\beta$-apo- $12^{\prime}$-carotenal from extractions of various samples accounted for $>95 \%$. Plasma triacylglycerol and total cholesterol were measured manually by using commercial in vitro enzymatic test kits (methods 334-UV and 401; Sigma).

Analysis and chromatography. Carotenoids were quantified by HPLC on a Hewlett Packard HPLC (model 1100; Waldbronn, Germany) as described by Breithaupt (2000), using a variable wavelength detector set to $450 \mathrm{~nm}$ and two mobile phases for carotenoid separation (methanolMTBE-water (81:15:4, by vol. (A); 6:90:4, by vol. (B)). The gradient was slightly modified to (time $(\mathrm{min}), \% \mathrm{~A}$ ): 0,$99 ; 39,44 ; 45,0 ; 50,99 ; 55,99$. The system for semipreparative HPLC was itemized by Breithaupt \& Bamedi (2001). LC-APcI-MS was run on a Micromass (Manchester, UK) VG platform II quadrupole MS equipped with an APcI interface, operating in the positive mode, as described by Breithaupt \& Schwack (2000). Within a running time of $55 \mathrm{~min}$, all major carotenoids (lutein, zeaxanthin, $\beta$-cryptoxanthin, $\alpha$-carotene, $\beta$-carotene, lycopene) and the internal standard ( $\beta$-apo-12'-carotenal) were baseline separated. The chromatogram matched the normal carotenoid distribution as described in the literature (Rock, 1997; Böhm \& Bitsch, 1999). To verify $\beta$-cryptoxanthin identification in human plasma, six plasma samples from group 1 (day 1) were pooled, cleaned up through a small glass column $(150 \times 10 \mathrm{~mm})$ packed with silica gel (according to the procedure described for $\beta$-cryptoxanthin ester isolation), the eluate concentrated to $200 \mu \mathrm{l}$ and analysed with LC-APcI-MS.

\section{Statistics}

Results are expressed as mean values and standard deviations. The areas under the curve (AUC $(0-24 \mathrm{~h})$ ) were determined automatically by the trapezoidal rule after subtraction of the fasting plasma $\beta$-cryptoxanthin concentrations. Likewise, plasma concentrations were corrected by the individual fasting $\beta$-cryptoxanthin concentrations. For comparison of the plasma concentrations and the AUC of the two groups and days, two-sided $F$ test and $t$ test were employed (each $P=0 \cdot 05$ ). All statistics were performed with Microsoft Excel 2000, Microsoft Corporation, Redmond, USA.

\section{Results}

All participants were instructed to consume a low-carotenoid diet for 2 weeks before and during the study in order to avoid falsification. Hence, fasting base concentrations of $\beta$-cryptoxanthin were only 0.06 (SD 0.05 ) (range $0.0-0 \cdot 2$ ) $\mu \mathrm{mol} / \mathrm{l}$ ( $n 12)$. Further characteristics of the participants (fasting plasma concentrations of cholesterol and triacylglycerol) on both intervention days are shown in Table 3 .
Table 3. Characteristics of the subjects (Mean values and standard deviations)

\begin{tabular}{|c|c|c|c|c|}
\hline & \multicolumn{2}{|c|}{ Day 1} & \multicolumn{2}{|c|}{ Day 2} \\
\hline & Mean & SD & Mean & SD \\
\hline Age (years)* & $27 \cdot 3$ & $5 \cdot 7$ & & \\
\hline BMl $\left(\mathrm{kg} / \mathrm{m}^{2}\right) \dagger$ & 24.5 & 3.0 & & \\
\hline \multicolumn{5}{|c|}{ Fasting plasma concentrations $†$} \\
\hline Total cholesterol (mmol/l) & 4.73 & 0.92 & 4.93 & 0.95 \\
\hline Triacylglycerol (mmol/l) & 1.09 & 0.58 & $1 \cdot 18$ & 0.59 \\
\hline$\beta$-Cryptoxanthin $(\mu \mathrm{mol} / /)$ & 0.06 & 0.05 & 0.05 & 0.04 \\
\hline
\end{tabular}

${ }^{*} n 12$ (six male and six female).

† $n 12$ (days 1 and 2 separately).

Fig. 1 shows two HPLC chromatograms illustrating the constitution of both supplements used on both days: chromatogram A shows the native carotenoid ester pattern of the purified papaya extract. Peak assignment was carried out by LC-APcI-MS analyses in the positive mode by evaluation of the respective quasimolecular ions $\left([\mathrm{M}+\mathrm{H}]^{+}\right)$, fragment ions and the absorption spectra. Besides $\beta$-cryptoxanthin caprate (peak 3), laurate (peak 4), myristate (peak 5) and palmitate (peak 6), a small amount of $\beta$-carotene (peak 2) was present, whereas nonesterified $\beta$-cryptoxanthin (peak 1) was totally absent. Efforts to reduce the $\beta$-carotene concentration by optimization of the open-column chromatography were not successful; the same is true for a minute amount of lycopene (peak 7). Since the concentrations of $\beta$-carotene and lycopene were comparatively low, both probably did not compete with excess $\beta$-cryptoxanthin and thus should not have affected absorption kinetics. Several minor peaks represented native cis-isomers of $\beta$-cryptoxanthin esters and were not studied in detail. Chromatogram B is of a saponified sample obtained after further purification by semi-preparative HPLC. In this supplement, only nonesterified $\beta$-cryptoxanthin (peak 1) was present.

Plasma carotenoids were determined using an HPLC system $(450 \mathrm{~nm})$ equipped with a $\mathrm{C}_{30}$ reversed-phase

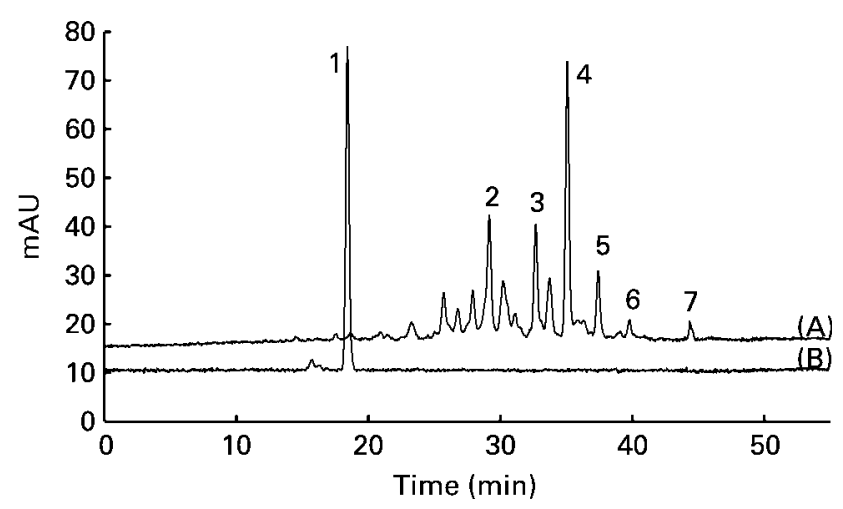

Fig. 1. Typical HPLC chromatograms $(450 \mathrm{~nm})$ of native papaya (Carica papaya L.) after clean-up by column chromatogaphy on silica gel $(A)$ and of a saponified sample (B). AU, absorbance units. Peak assignment was done by analyses with liquid chromatography-atmospheric pressure chemical ionization-MS as follows: peak 1, non-esterified all-trans- $\beta$-cryptoxanthin; peak 2 , $\beta$-carotene; peak $3, \beta$-cryptoxanthin caprate; peak $4, \beta$-cryptoxanthin laurate; peak $5, \beta$-cryptoxanthin myristate; peak $6, \beta$-cryptoxanthin palmitate; peak 7 , lycopene. For details of procedures, see p. 797. 


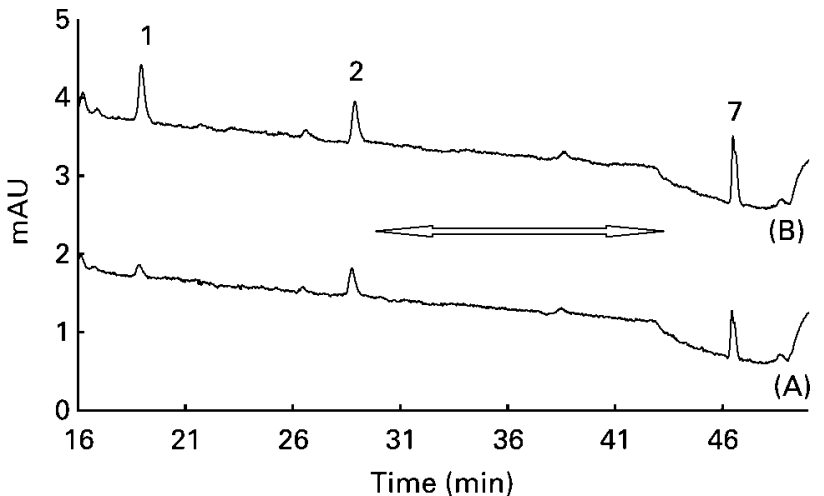

Fig. 2. Typical HPLC chromatograms (extended sections; $450 \mathrm{~nm}$ ) of plasma samples (group 1, day 1) obtained from one subject $0(A)$ and 24 (B) h after consumption of esterified $\beta$-cryptoxanthin. Peak assignment: peak 1 , non-esterified all-trans- $\beta$-cryptoxanthin; peak 2 , $\beta$-carotene; peak 7 , lycopene. AU, absorbance units; $\Leftrightarrow$, retention time window in which $\beta$-cryptoxanthin esters appear. For details of subjects and procedures, see Table 3 and p. 797.

column. Confirmation of carotenoids in human plasma by LC-MS has been described earlier by Khachik et al. (1992), who used a nitrile-bonded column for separation. $\beta$-Cryptoxanthin was unequivocally identified by its u.v.visible spectrum $(420-452-478 \mathrm{~nm}$; diode array detector) and its quasimolecular ion $\mathrm{m} / \mathrm{z} \quad 553[\mathrm{M}+\mathrm{H}]^{+}$in a pooled sample. Typical examples of HPLC chromatograms (extended sections) obtained from the plasma of one individual (group 1, day 1) before and after ingestion of esterified $\beta$-cryptoxanthin are presented in Fig. 2. However, neither HPLC nor LC-APcI-MS analyses gave evidence for the presence of $\beta$-cryptoxanthin esters in plasma. Minor peaks in the time window indicated were also present in fasting plasma samples and could not be unequivocally allocated to $\beta$-cryptoxanthin esters. The absence of $\beta$-cryptoxanthin esters is in accordance with the results of Wingerath et al. (1995), who also were not able to detect these compounds either in serum or in chylomicrons.

For quantitative assessment, the average concentrations of $\beta$-cryptoxanthin $v$. the time of sampling were plotted (Fig. 3). Independently of spiking the diets with esterified or non-esterified $\beta$-cryptoxanthin, the plasma concentration of all participants increased significantly $(\mathrm{P}<0.05)$ after consumption of $\beta$-cryptoxanthin-enriched yoghurts. Concentration curves were statistically not distinguishable according to two-sided $F$ test and $t$ test $(P<0.05)$ applied to both groups. The absorption maxima in the plasma were reached between 6 and $12 \mathrm{~h}$ after supplementation at both intervention days. The maximum plasma value measured in an individual in the study was $205 \mathrm{nmol}$ $(113 \mu \mathrm{g}) / \mathrm{l}$; this value occurred after supplementation with esterified $\beta$-cryptoxanthin.

In order to account for potential variations in the blood total triacylglycerol or cholesterol levels, all $\beta$-cryptoxanthin concentrations were corrected for both triacylglycerol and cholesterol. Each $\beta$-cryptoxanthin value was divided by the corresponding triacylglycerol (or cholesterol) concentration and multiplied by the mean triacylglycerol (or cholesterol) concentration of all participants. As an example, $\beta$-cryptoxanthin plasma responses adjusted for triacylglycerol are shown in Fig. 4. Standardization for total cholesterol (results not shown) revealed the same results.

To compare the absorption behaviour for esterified and non-esterified $\beta$-cryptoxanthin, a summarized value comprising all sampled points, not only the maxima, is required. This problem can be solved by evaluation of the AUC, calculated from the appropriate figure (plasma concentration of $\beta$-cryptoxanthin $v$. time of sampling). Furthermore, the AUC is proportional to the bioavailability (Yao et al. 2000), hence allowing an estimation of how well either of the two forms are resorbed. Mean AUC and standard deviations were calculated for days 1 and 2 and groups 1 and 2. This proved AUC of the groups not to be distinguishable, either within one group, or between the two groups (two-sided $F$ test and $t$ test, $P=0.05$ ). Therefore, AUC were combined for days 1 and 2, summing up total participants according to the $\beta$-cryptoxanthin forms ingested. This resulted in mean AUC of: esterified $\beta$-cryptoxanthin 0.91 (SD 0.98$) \mu \mathrm{mol} / \mathrm{h}$ per litre $(n 12)$; non-esterified $\beta$-cryptoxanthin 1.09 (SD 0.82) $\mu \mathrm{mol} / \mathrm{h}$ per litre $(n 12)$. This demonstrates again that AUC were not distinguishable. Thus, plasma concentrations of $\beta$-cryptoxanthin were comparable, even with regard to AUC,
(A)

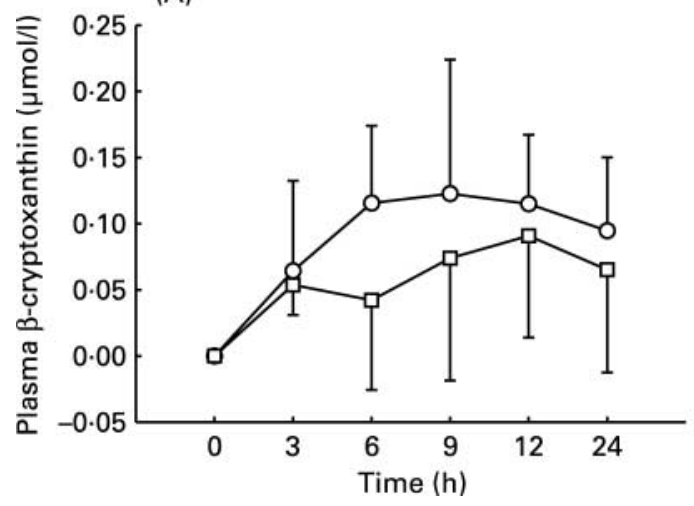

(B)

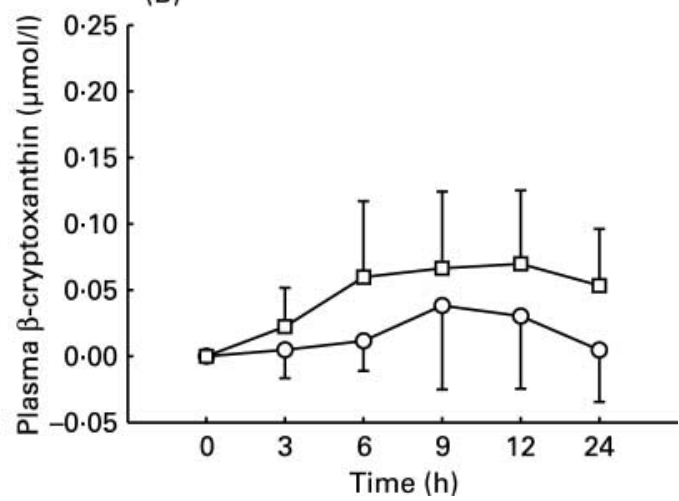

Fig. 3. Dependence of plasma $\beta$-cryptoxanthin concentrations on sampling time after a single dose of esterified $\beta$-cryptoxanthin $(O)$ or nonesterified $\beta$-cryptoxanthin $(\square ; 1.3 \mathrm{mg}$ each). (A), day 1 ; (B), day 2 . Values are means with standard deviations shown by vertical bars (twelve per group). For details of subjects and procedures, see Table 3 and p. 797. 
(A)

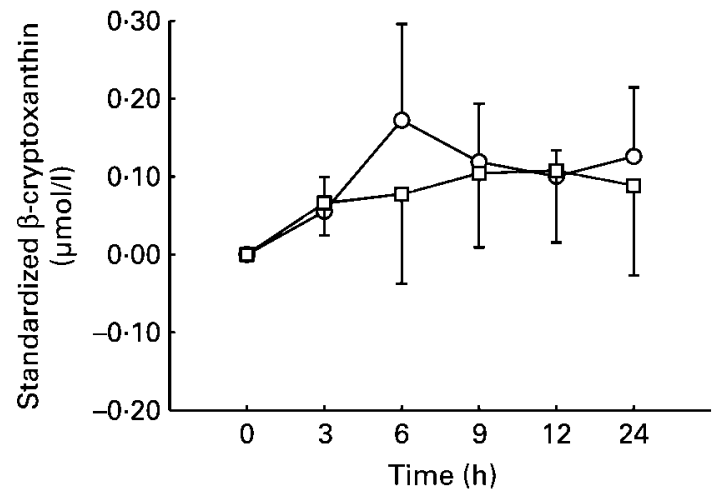

(B)

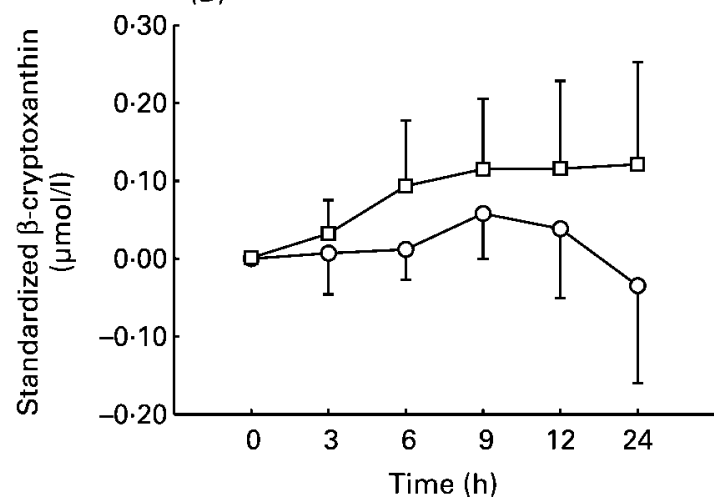

Fig. 4. Plasma $\beta$-cryptoxanthin concentrations, standardized to the plasma triaclyglycerol concentration, after a single dose of esterified $\beta$-cryptoxanthin $(O)$ or non-esterified $\beta$-cryptoxanthin $(\square ; 1.3 \mathrm{mg}$ each). (A), day 1 ; (B), day 2 . Values are means with standard deviations shown by vertical bars (twelve per group). For details of subjects and procedures, see Table 3 and p. 797.

whether the subjects were given esterified or non-esterified $\beta$-cryptoxanthin.

\section{Discussion}

Although it is presumed that dietary $\beta$-cryptoxanthin esters are hydrolysed in the intestine before absorption, results are not yet available comparing the plasma concentrations achieved after ingestion of esterified or non-esterified forms. Consequently, a crossover study was designed using esterified or non-esterified $\beta$-cryptoxanthin from a natural source, applied as one single dose together with a balanced breakfast. Fasting base concentrations of $\beta$-cryptoxanthin given in the literature usually range between 0.2 and $0.5 \mu \mathrm{mol} / 1 \mathrm{l}(0.21 \mu \mathrm{mol} / \mathrm{l}$, Krinsky et al. 1990; $0.48 \mu \mathrm{mol} / \mathrm{l}$, Grobusch-Klipstein et al. 2000). In a recent publication, $\beta$-cryptoxanthin levels in fasting sera of subjects from five European countries ranged from 0.14 (Northern Ireland, UK) to $0.40 \mu \mathrm{mol} / \mathrm{l}$ (Spain) (Olmedilla et al. 2001). Thus, the fasting base concentrations of $\beta$-cryptoxanthin were well below the usually observed concentration in human plasma, and demonstrated the effect of the depletion phase before beginning the intervention. Both triacylglycerol and total cholesterol concentrations were in the normal range for all subjects in both groups (desirable concentrations: triacylglycerol $<1.25 \mathrm{mmol} / \mathrm{l}$, cholesterol $<5.18 \mathrm{mmol} / \mathrm{l}$; Jordan et al. 1995).

After ingestion of $\beta$-cryptoxanthin-rich diets, absorption maxima in the plasma were reached between 6 and $12 \mathrm{~h}$ after application. This is in accordance with the observations of Wingerath et al. (1995), who found serum $\beta$-cryptoxanthin concentrations increasing up to $9 \mathrm{~h}$ after ingestion of tangerine juice concentrate. The maxima probably represent a transient increase in chylomicron-associated $\beta$-cryptoxanthin. A second peak, usually observed in plasma $\beta$-carotene studies after $24-48 \mathrm{~h}$ and representing the increase in the LDL fraction (Rock, 1997), was not observed during the study period of $24 \mathrm{~h}$. Thus, each of the curves showed only one maximum. Since concentration curves were statistically not distinguishable, bioavailability of both non-esterified $\beta$-cryptoxanthin and a native mixture of different $\beta$-cryptoxanthin esters were comparable in our present study.

The maximum plasma $\beta$-cryptoxanthin concentration obtained was $205 \mathrm{nmol}(113 \mu \mathrm{g}) / \mathrm{l})$. Based on the assumption that about $4 \%$ of the body weight is plasma (2.4 litres plasma $/ 60 \mathrm{~kg}$; Barua, 1999), this concentration corresponds to an absolute amount of $271 \mu \mathrm{g} \beta$-cryptoxanthin, amounting to approximately one-quarter of the applied dose. This is in accordance with a relatively low efficiency of carotenoid absorption, reported to range from $5 \%$ for raw vegetables up to $50 \%$ or more for micellar solutions (Olson, 1994). Most of the carotenoid might have been metabolized to vitamin A (which was not studied here) or excreted through the gastrointestinal tract. However, a significant increase in $\beta$-cryptoxanthin plasma concentrations was observed for all participants. After consuming esterified $\beta$-cryptoxanthin, a higher variability in plasma responses was observed (Fig. 3). Although the definite reason for this effect is unknown, a possible explanation could be different enzyme activity of individual participants. Remarkably, the mean plasma $\beta$-cryptoxanthin concentrations on the second day seemed to show a lower increase (Fig. 3). Applying statistical methods (two-sided $F$ test and $t$ test, $P=0.05$ ) proved both data sets not to be distinguishable, showing that there is in fact no difference in the absorption characteristics of both days. Yet we are of the opinion that the statistical result is not to be overestimated, since the deviation of the plasma concentrations of the participants is relatively high. Upon this fact, the base concentrations of days 1 and 2 were compared and were found to be similar; thus, a lessened uptake due to plasma 'saturation' is not very likely. It can be assumed that the time frame of 2 weeks between the two experimental days was not sufficient to ensure the same metabolic state as at the beginning of the study. The reason for this behaviour is as yet unknown and requires further investigation.

Carotenoids are transported in association with lipoproteins, so plasma triacylglycerol and cholesterol concentrations are usually correlated with circulating carotenoid concentrations (Thurnham, 1989). As plasma triacylglycerol depends on the diet consumed on the intervention day, which was not controlled by the present study, a 
high additional fat intake may result in a lowered plasma carotenoid response. Thus, plasma $\beta$-cryptoxanthin concentrations were corrected for both total triacylglycerol and cholesterol (Fig. 4). The shape of the resulting curves is close to that of the unadjusted data set, pointing out that standardization did not change the overall results when performed. One exception is the standard deviation corresponding to values obtained $6 \mathrm{~h}$ after the intervention. This is due to high individual deviations in the triacylglycerol deviation of one single person. Further on, standardization to triacylglycerol demonstrates that the plasma $\beta$-cryptoxanthin response is not influenced by triacylglycerol consumed by the participants during the intervention days.

Quantitatively, there was no difference in the resulting plasma response when the subjects were given esterified or non-esterified $\beta$-cryptoxanthin. Since $\beta$-cryptoxanthin was administered in its esterified or non-esterified form, resulting in comparable plasma concentrations, it may be concluded that there is no preferential uptake of the non-esterified form, as discussed by Wingerath et al. (1995). In fact, the present results support the existence of an effective enzymatic cleavage system in the gut lumen, accepting different natural $\beta$-cryptoxanthin esters as substrates.

\section{Acknowledgement}

We thank all volunteers of the Institute of Food Science, University of Hannover, Germany, who participated in this study.

\section{References}

Barua AB (1999) Intestinal absorption of epoxy- $\beta$-carotenes by humans. Biochem J 339, 359-362.

Bieri JG, Brown ED \& Smith JC Jr (1985) Determination of individual carotenoids in human plasma by high performance liquid chromatography. J Liq Chromatogr 8, 473-484.

Böhm V \& Bitsch R (1999) Intestinal absorption of lycopene from different matrices and interactions to other carotenoids, the lipid status and the antioxidant capacity of human plasma. Eur J Nutr 38, 118-125.

Breithaupt DE (2000) Enzymatic hydrolysis of carotenoid fatty acid esters of red pepper (Capsicum annuum L.) by a lipase from Candida rugosa. Z Naturforsch 55C, 971-975.

Breithaupt DE \& Bamedi A (2001) Carotenoid esters in vegetables and fruits: a screening with emphasis on $\beta$-cryptoxanthin esters. J Agric Food Chem 49, 2064-2070.

Breithaupt DE, Bamedi A \& Wirt U (2002) Carotenol fatty acid esters: easy substrates for digestive enzymes? Comp Biochem Physiol B 134, 721-728.

Breithaupt DE \& Schwack W (2000) Determination of free and bound carotenoids in paprika (Capsicum annuum L.) by LC/ MS. Eur Food Res Technol 211, 52-55.

Gärtner C, Stahl W \& Sies H (1997) Lycopene is more bioavailable from tomato paste than from fresh tomatoes. Am J Clin Nutr 66, 116-122.

Goswami UC (1984) Metabolism of cryptoxanthin in freshwater fish. Br J Nutr 52, 575-581.
Grobusch-Klipstein K, Launer LJ, Geleijnse JM, Boeing H, Hofman A \& Witteman JCM (2000) Serum carotenoids and atherosclerosis: the Rotterdam study. Atherosclerosis 148, 49-56.

Ito Y, Ochiai J, Sasaki R, et al. (1990) Serum concentrations of carotenoids, retinol, and $\alpha$-tocopherol in healthy persons determined by high-performance liquid chromatography. Clin Chim Acta 194, 131-144.

John J, Kishore GS, Subbarayan C \& Cama HR (1970) Metabolism and biological potency of cryptoxanthin in rat. Indian $J$ Biochem 7, 222-225.

Jordan P, Brubacher D, Moser U, Stähelin HB \& Gey KF (1995) Vitamin $\mathrm{E}$ and vitamin A concentrations in plasma adjusted for cholesterol and triacylglycerides by multiple regression. Clin Chem 41, 924-927.

Khachik F, Beecher GR \& Goli MB (1992) Separation and identification of carotenoids and their oxidation products in the extracts of human plasma. Anal Chem 64, 2111-2122.

Khachik F, Spangler CJ \& Smith Jr JC (1997) Identification, quantification, and relative concentrations of carotenoids and their metabolites in human milk and serum. Anal Chem 69, 1873-1881.

Krinsky NI, Russett MD, Handelman J \& Snodderly M (1990) Structural and geometrical isomers of carotenoids in human plasma. J Nutr 120, 1654-1662.

Olmedilla R, Granado F, Gil-Martinez E, Blanco I \& RojasHidalgo E (1997) Reference values for retinol, tocopherol, and main carotenoids in serum of control and insulin-dependent diabetic Spanish subjects. Clin Chem 43, 1066-1071.

Olmedilla B, Granado F, Southon S, et al. (2001) Serum concentrations of carotenoids and vitamins $\mathrm{A}, \mathrm{E}$, and $\mathrm{C}$ in control subjects from five European countries. Br J Nutr 85, 227-238.

Olson JA (1994) Absorption, transport, and metabolism of carotenoids in humans. Pure Appl Chem 66, 1011-1016.

Rock CL (1997) Carotenoids: biology and treatment. Pharmacol Ther 75, 185-197.

Ross MA, Crosley LK, Brown KM, et al. (1995) Plasma concentrations of carotenoids and antioxidant vitamins in Scottish males: influences of smoking. Eur J Clin Nutr 49, 861-865.

Scherz H, Senser F \& Souci SW (2000) Souci-FachmannKraut: Die Zusammensetzung der Lebensmittel: NährwertTabellen (Food Composition and Nutrition Tables), 6th ed. Stuttgart: Medpharm Scientific Publishers, Wissenschaftliche Verlagsgesellschaft $\mathrm{mbH}$.

Stahl W, Schwarz W, Sundquist AR \& Sies H (1992) cis-trans Isomers of lycopene and $\beta$-carotene in human serum and tissues. Arch Biochem Biophys 294, 173-177.

Thurnham DI (1988) Do higher vitamin A requirements in men explain the difference between the sexes in plasma provitamin A carotenoids and retinol? Proc Nutr Soc 47, 181A.

Thurnham DI (1989) Lutein, cholesterol, and risk of cancer. Lancet 19, 441-442.

Yao L, Liang Y, Trahanovsky WS, Serfass RE \& White WS (2000) Use of a $13 \mathrm{C}$ tracer to quantify the plasma appearance of a physiological dose of lutein in humans. Lipids 35, 339-348.

van Vliet T, Schreurs WHP \& van den Berg H (1995) Intestinal $\beta$-carotene absorption and cleavage in men: response of $\beta$-carotene and retinyl esters in the triacylglycerol-rich lipoprotein fraction after as single oral dose of $\beta$-carotene. Am J Clin Nutr 62, 110-116.

Wingerath T, Stahl W \& Sies H (1995) $\beta$-Cryptoxanthin selectively increases in human chylomicrons upon ingestion of tangerine concentrate rich in $\beta$-cryptoxanthin esters. Arch Biochem Biophys 324, 385-390. 